\title{
Trayectorias Organizacionales y Empoderamiento Comunitario: Un Análisis de Interfaz en Dos Localidades de la Región de la Araucanía
}

\section{Organizational Trajectories and Community Empowerment: An Interface Analysis in Two Communities of the Araucanía Region}

\author{
Alba Zambrano, Gonzalo Bustamante y Mauricio García \\ Universidad de La Frontera
}

\begin{abstract}
Se reportan los principales resultados de una investigación cuyo propósito fue indagar sobre las variables psico-socio-culturales presentes en la interfaz entre organizaciones comunitarias de base y agentes públicos que potencian o restringen procesos de empoderamiento organizacional y comunitario. Se empleó una metodología cualitativa, apoyada por el análisis estructural de redes, analizándose en 2 localidades de la región de la Araucanía 4 tipos de organizaciones comunitarias. Los resultados muestran que en las localidades predomina un interfaz de tipo semiclientelar, clientelar y paternalista, formas de relación que se centran en la entrega de recursos por parte del municipio para resolver algunas necesidades inmediatas de la comunidad, tendiendo a predominar en las organizaciones objetivos inmediatos y una participación centrada en conseguir estos recursos.
\end{abstract}

Palabras clave: empoderamiento, enfoque de interfaz, desarrollo comunitario.

The main findings of a study that investigated the psychosocial cultural factors affecting the interface between community organizations and public agencies are reported. Four types of organizations are analyzed in 2 different communities, using a qualitative methodology and a structural networks analysis. In both communities the paternalistic, client and semi-client interface type predominate. These types of relationships focus on the delivery of resources by the municipal institution as a way of addressing the immediate needs of the community. In turn, the community organizations center their efforts on short-term objectives and participation aimed at obtaining these resources.

Keywords: empowerment, interface approach, community development.

Introducción

$\mathrm{Al}$ analizar la literatura y observar lo que ocurre en el campo de la intervención social, se puede evidenciar una tendencia a fomentar la incorporación de la comunidad como un actor y referente relevante en las intervenciones y políticas sociales. Ello se vincula con un intento por dar respuesta a profundas y variadas transformaciones acontecidas en la sociedad en el plano político y económico, de la sociabilidad y subjetividad, entre otros (Llena \& Úcar, 2006; Zambrano, 2007).

En este marco la acción comunitaria tiene por propósito revitalizar la sociabilidad, aportando a la vinculación y organización social, como también a los emprendimien-

Alba Zambrano Constanzo, Departamento de Psicología, Universidad de La Frontera, Temuco, Chile.

Gonzalo Bustamante Rivera, Departamento de Psicología, Universidad de La Frontera, Temuco, Chile.

Mauricio García Ojeda, Departamento de Ciencias Sociales e Instituto de Desarrollo Local y Regional, Universidad de La Frontera, Temuco, Chile.

La correspondencia relativa a este artículo debe ser dirigida a la Dra. Alba Zambrano Constanzo, Departamento de Psicología, Universidad de La Frontera, Montevideo 0830, Temuco, Chile. E-mail: albaz@ufro.cl

La investigación fue financiada por la Dirección de Investigación y Desarrollo de la Universidad de la Frontera (DIDUFRO 120613).

Colaboraron en la investigación los estudiantes o tesistas: María Antonieta Campos, Iván Neira, Felipe Vega y Silvia Alarcón. Se agradece la participación de las organizaciones territoriales, comunitarias y estudiantiles que colaboraron en el estudio. 
tos colectivos para mejorar las condiciones de vida, especialmente en población socialmente desfavorecida (Zambrano, 2007). En esta perspectiva, el grupo y su organización aparece como un elemento que, dentro de un espacio social determinado, permite redensificar la vida social mediante el fortalecimiento del sujeto político, promoviendo estructuras de relaciones que hacen posible la participación democrática (Lapalma, 2001; Montero, 2005).

La psicología comunitaria ha venido focalizando parte importante de su trabajo en atender los procesos y variables vinculadas al desarrollo de la comunidad, tomando como uno de sus ejes centrales de estudio la constitución y fortalecimiento de las organizaciones como instancias articuladoras entre la institucionalidad y la comunidad (Rozas, 1995). La participación, las relaciones entre actores diversos, la generación de liderazgos, la organización, formación y fortalecimiento de redes sociales, la identidad comunitaria, el empoderamiento (empowerment en inglés), el soporte psicosocial y el sentimiento de comunidad son algunos temas abordados desde este campo de la psicología.

En Chile, aunque las llamadas dimensiones intangibles de los problemas sociales son incorporadas en la política social de un modo más explícito a partir de la década de los noventa, valorando de un modo creciente la subjetividad y dimensiones relativas a la participación y a la vida en comunidad (Alfaro, 2000), queda de manifiesto la utilización de lógicas de intervención que producen desencuentros y dificultades con los principios que orientan la acción comunitaria (Alfaro \& Zambrano, en prensa; Krause \& Jaramillo,1998; Saavedra, 2005; Serrano, 2005).

La Araucanía es una de las regiones con los peores indicadores de desarrollo humano en Chile y, en consecuencia, se han ejecutado múltiples programas y proyectos con financiamiento estatal orientados a mejorar esta condición. En este contexto resulta relevante preguntarse acerca de la capacidad de las organizaciones comunitarias y agentes de desarrollo para favorecer procesos de fortalecimiento comunitario que colaboren en mejorar la calidad de vida de las perso- nas involucradas en estas iniciativas.

En consideración a lo anterior, en este artículo se analiza, desde un enfoque de interfaz, las trayectorias de las organizaciones comunitarias de base de dos localidades de la región de la Araucanía, con el fin de descubrir los factores endógenos y exógenos más relevantes que se asocian a experiencias de empoderamiento comunitario, identificando los principales obstaculizadores en dicho proceso. En segundo lugar, se proponen líneas de acción para fortalecer dinámicas de empoderamiento organizacional y comunitario en las localidades estudiadas.

\section{Marco Teórico}

\section{Aportes del Enfoque del Empoderamiento a la Intervención Social}

En los modelos tradicionales de intervención social es el operador social quien presume poseer la experticia necesaria para determinar la naturaleza del problema y el tratamiento que conviene aplicar, relegando a las personas implicadas al rango de simples ejecutantes. Este tipo de intervención trata a los beneficiarios de estos servicios como individuos carentes de competencia para hacerse cargo de su realidad y refuerza la falta de poder de estos sobre las propias circunstancias y condiciones de vida (Le Bossé \& Dufort, 2002; Perkins, 1995). Esta postura propicia que la responsabilidad del cambio quede depositada en los agentes de desarrollo, quienes conducen el proceso, limitando consecuentemente la asunción de control de la comunidad respecto de su situación (Martínez, 2006; Montero, 1991; Pérez-Luco, 2003).

Numerosos autores han desarrollado perspectivasy prácticas sociales alternativas a este enfoque (De Paula, 2003; Durston, Duhart, Miranda \& Monzó, 2005; Lipszyc, 1993; Plough \& Olafson, 1994). Entre estas, la intervención centrada en el desarrollo del empoderamiento de las personas y de las comunidades aparece como una de las más pertinentes (Le Bossé \& Dufort, 2002; Newbrough, 1992; Silva \& Martínez, 2004).

Este enfoque permite dar respuesta a cuestiones fundamentales en la interven- 
ción social. Por una parte, pone énfasis en las fortalezas de las personas, promoviendo una nueva imagen de ellas como ciudadanos con derechos y opciones. Define también una nueva aproximación a la relación de ayuda, que sitúa a los profesionales como colaboradores que trabajan con miembros de la comunidad en un afán por cambiar su mundo social, superando la visión tradicional de expertos alejados del mundo de las personas (Rappaport, 1981). Adicionalmente, destaca un aspecto gravitante en la transformación social, como son las relaciones de poder. Desde este enfoque se enfatiza que la relación desigual entre las personas y grupos en la distribución y acceso a recursos de diversa índole incide en el control de las personas sobre la propia realidad, mediatizando, por tanto, las posibilidades de desarrollo humano (Zambrano, 2007).

En la lengua inglesa la palabra empowerment es un término común que puede ser empleado en múltiples contextos para significar un aprovechamiento positivo del poder. Desde el punto de vista lingüístico, esta expresión puede ser separada en tres elementos. El primero es el radical power que significa poder en inglés, el segundo es el prefijo em, que, agregado al radical power, expresa un movimiento de acceso al poder. La asociación de estos dos elementos forma el verbo empower, que designa generalmente a un aumento del poder. El último elemento es el sufijo ment que sugiere la presencia de un estado resultante de aumento del poder. En un primer momento, empowerment puede, entonces, ser comprendido como un movimiento (proceso) general de adquisición de poder en vistas a alcanzar un objetivo preciso (Le Bossé \& Dufort, 2002).

Rappaport (1987) definió empoderamiento como "un proceso, un mecanismo mediante el cual las personas, organizaciones y comunidades logran control sobre sus asuntos" (p. 122). El autor asume un punto de vista ecológico, señalando el carácter socialmente complejo y transversal del empoderamiento. Así, señala que para el caso del empoderamiento comunitario se pueden identificar dos dimensiones o componentes complementarios: uno se centra en la autodeterminación personal, que sería la capa- cidad de determinar la propia vida (sentido de competencia personal); el otro se centra en la determinación social que se refiere a la posibilidad de participación democrática (sentido de competencia comunitaria).

El empoderamiento es en gran medida un intento por extender el campo de las acciones posibles tanto desde el punto de vista de los recursos personales (e.g., habilidades en la comunicación, liderazgo) como los del entorno (e.g., acceso a servicios adecuados, a una vivienda conveniente). Se trata de un poder para atraer el cambio deseado o contribuir a ello (Le Bossé \& Dufort, 2002).

Algunos autores han criticado el uso de este concepto por presentar varias limitaciones conceptuales y prácticas (Gil, 2003; Montero, 2003; Vásquez, 2004). Por ejemplo, se ha señalado que el concepto puede conducir al error de creer que los "agentes externos" que tienen poder pueden transferirlo automáticamente a quienes carecen de él. Sin embargo, si se considera el sentido original del concepto de empoderamiento en la comprensión otorgada por Le Bossé y Dufort (2002), queda claro que el poder no se transfiere sino que se desarrolla en un proceso complejo. Desde una posición generativa del poder, este se construye, se gana -a veces a costa de fuertes conflictos- y la labor del agente de desarrollo es acompañar en ese proceso a las personas, poniendo a disposición su propio poder. La acción de un agente externo es constituirse en colaborador porque, como lo indica Taliferro (1991), el verdadero poder no puede otorgarse sino que se desarrolla desde "adentro". Más aún, como lo muestra la práctica, en un real empoderamiento el proceso toma direcciones imprevistas, en donde es claro que el control no está en manos del agente externo (Rowlands, 1997).

$\mathrm{Al}$ atender a las relaciones de poder, el empoderamiento pone de relieve la asimetría entre distintos actores sociales (Craig \& Mayo, 1994; Friedmann, 1992; Rappaport, 1987; Rowlands 1997; Zimmerman, 1995). Especialmente aquellos autores que trabajan con grupos en mayor desventaja señalan que el empoderamiento es el proceso mediante el cual se trata de generar mecanismos que disminuyan dicha asimetría, tratando de revertir las relaciones de poder a favor 
de quienes están en desventaja, para equilibrar la situación (Gil, 2003; Rowlands, 1997; Serrano-García \& López, 1994).

Para Zimmerman (2000) el esfuerzo de ejercer control sobre las contingencias de las propias conductas o las conductas colectivas es central pero no suficiente para señalar que hay empoderamiento; otros componentes, como la acción colectiva, la conciencia crítica y la movilización de recursos, son también necesarios. Estos aspectos son posibles de encontrar en los niveles individual, organizacional y comunitario. Según este autor, para lograr el empoderamiento en el nivel individual son básicos la participación con otros, lograr metas, los esfuerzos para generar acceso a los recursos y una comprensión crítica del medio sociopolítico. En el nivel organizacional el empoderamiento incluye procesos organizacionales y estructuras que animan la participación de los miembros y estimulan la efectividad organizacional. Por último, a nivel comunitario el empoderamiento se refiere a las acciones colectivas para mejorar las condiciones de vida y las conexiones entre organizaciones de la comunidad y entre estas y otras instancias o agencias. En esta perspectiva, una comunidad es competente cuando sus integrantes poseen las habilidades, deseos y recursos para implicarse en actividades que mejoran la vida de la comunidad (Zimmerman, 2000).

Un escenario comunitario que favorezca el empoderamiento debe reunir ciertas condiciones. Maton (2008) propone seis características: un sistema de creencias basado en el grupo, actividades centrales, ambiente que favorezca las relaciones, estructura de oportunidad para el ejercicio de roles, liderazgo y, por último, cambio y mantención del escenario.

\section{Desarrollo Local Endógeno y Trabajo Comunitario}

Un conjunto de teorías, desde diversas disciplinas (economía, antropología, sociología, entre otras), concuerdan en cuestionar el paradigma que asocia el desarrollo con variables solo económicas e incluyen variables de tipo sociocultural que, junto con las económicas, permitirían comprender y dirigir intencionadamente procesos de desarrollo.

Desde estas teorías, se concuerda en que el desarrollo local endógeno rompe con el positivismo clásico de tipo lineal y monocausal, para comenzar a utilizar una perspectiva más sistémica y compleja. Asume una mirada del desarrollo como un proceso "de abajo hacia arriba", en el que son relevantes las decisiones de sus actores internos (Bustamante, 2003).

El carácter endógeno del desarrollo radica en que los sujetos del desarrollo son necesariamente los actores que pertenecen a un territorio, además de otros actores externos al territorio pero internos al proceso, como el actor institucional-público. También alude a que los recursos (sociales, humanos, capital social, subjetivos, materiales) provienen del territorio y que las decisiones de las acciones tendientes al desarrollo orientado al mejoramiento de las condiciones de vida provienen de los actores locales (Bustamante, 2003).

Desde este marco, componentes psicosocioculturales, tales como el capital social, las relaciones, liderazgos, participación, empoderamiento en sus distintos niveles, son recursos claves que en interacción con otros recursos pueden propiciar circuitos virtuosos de desarrollo (Boisier, 1999, 2001, Noviembre; Durston, 2002; Kliksberg, 1999; Moncayo, 2001; Vásquez-Barquero, 1999). Tanto las instituciones y organizaciones como los agentes privados y públicos deben aprender a interrelacionarse y avanzar en construir un proyecto común (Marchioni, 2001).

El desarrollo comunitario, como lo proponen Blanco y Rebollo (2002), sería un proceso que busca la transformación de un territorio, la que puede ser de diversa índole: social, económica o urbanística, entre otras. En este proceso juegan un rol de suma relevancia tanto la organización de la población o la constitución de un grupo o grupos en torno a un proyecto común, como la participación, pues se trataría que la gente se fuera implicando de un modo creciente en iniciativas que le son relevantes (Barbero \& Cortés, 2005; Marchioni, 2001).

La organización comunitaria se refiere a grupos organizados de personas que 
comparten intereses para cohesionar e integrar a los habitantes en torno a propósitos comunes (Carballeda, 2002). Para poder comprender la estructura y funcionamiento de estas organizaciones es necesario comprender y desentrañar las relaciones que en ellas se generan, incluyendo la historia, las dinámicas relacionales, las estructuras organizacionales y las formas de liderazgo, todos ellos comprendidos como procesos y no como elementos estáticos (Shein, 1998).

\section{Sistemas Socioculturales Locales: Capital Social, Empoderamiento e Interfaz}

Las teorías del capital social han aportado la inclusión de variables socioculturales en el análisis de experiencias de desarrollo, específicamente respecto de la capacidad que tienen los emprendimientos asociativos basados en la colaboración y confianza para el logro de metas comunes (Durston, 2002; Roberts, 2001, Diciembre).

El concepto capital social, siguiendo a Durston (1999), hace "referencia a normas, instituciones y organizaciones que promueven la confianza y la cooperación, entre las personas, las comunidades y en la sociedad en su conjunto" (p. 6). Es a través de la repetición de situaciones de confianza, en el marco de relaciones enmarcadas en normas, instituciones y organizaciones, que la cooperación puede fomentarse, pudiendo adquirir un sentido pragmático si se considera que los diversos actores pueden evaluar que para lograr mejor sus estrategias deben cooperar en función de sus objetivos comunes (Barreiro, 2002; Durston, 2002). Existen varias formas o tipos de capital social: individual, grupal, comunitario, de puente y de escalera (Dirven, 2001, Septiembre; Durston, 2002; Flores \& Rello, 2001, Septiembre). Específicamente, el capital social comunitario es entendido como aquel capital social que reside "en las estructuras que forman la institucionalidad de cooperación comunitaria, en sus estructuras de gestión y acción" (Durston, 2002, p. 41).

Se puede apreciar una estrecha relación entre los conceptos de empoderamiento en el nivel comunitario con el de capital social comunitario. Ambos aluden a la relevancia de los recursos humanos en la forma de re- des asociativas que pueden brindar apoyo o colaborar en los propósitos de la comunidad. Así, la propiedad compartida de esta red permitiría tener acceso a otro tipo de recursos que son de importancia para las personas en la medida que pueden aportar a mejorar las condiciones de vida. Pero, mientras el capital social pone el acento sobre el recurso que reporta esta red y los atributos de la misma (confianza, reciprocidad, trabajo en torno a propósitos comunes), el empoderamiento lo pondría sobre el proceso de acceso a estos recursos y como ello implica obtener mayores grados de autodeterminación.

Desde los enfoques estructurales de redes presentes en las teorías del capital social, se considera que los vínculos existentes, concebidos como redes sociales en tanto fuentes de capital social, son uno de los factores endógenos asociados a las experiencias de empoderamiento comunitario, aportando, además, a la comprensión de las trayectorias de las organizaciones comunitarias desde un enfoque de interfaz (García, 2005). Específicamente, en este estudio se emplean dos niveles de análisis: la estructura general de las redes y la posición e influencia que tienen los integrantes de las estructuras reticulares.

El enfoque de interfaz destaca el análisis de las relaciones en la zona de intercambio entre el capital social de la comunidad y otros sistemas (como el municipal). Estas interacciones concretas, como las lógicas en juego entre los actores (colaboración versus confrontación), pueden tener implicancias positivas o negativas para la comunidad. Este enfoque explora "cómo las discrepancias de interés social, interpretación cultural, conocimiento y poder son intermediados, perpetuados o transformados en puntos críticos de confrontación y cooperación" (Long, 1999, p. 21). La interfaz corresponde a "la zona de intercambio entre dos o más sistemas" (Durston, 2002, p. 43) que, en el caso de las vinculaciones verticales, corresponden principalmente al Estado con sus programas y agentes y sus relaciones concretas con las comunidades.

A partir de su trabajo de campo, Durston (2002) desarrolla una tipología para describir los tipos de interacción entre el capital 
social y los organismos públicos en la zona de interfaz. Esta tipología corresponde a un continuo que va desde (a) clientelismo autoritario, represivo y/o cleptocrático, (b) clientelismo pasivo, (c) semiclientelismo, (d) organismos "empoderadores" y apoyadores hasta (e) sinergia. El clientelismo autoritario se caracteriza por reprimir con violencia el capital social comunitario. El pasivo opera transformando el capital social comunitario en recepción pasiva de recursos, generando dependencia. El semiclientelismo fomenta la organización de la comunidad, su capacidad de generar propuestas y la protege de la interferencia negativa externa. Los organismos "empoderadores" profundizan el semiclientelismo, ampliando su radio de acción y fortaleciendo a los actores sociales débiles. La sinergia se caracteriza por comunidades que gestionan sus propias estrategias, establecen contratos con el Estado y otros organismos, gestionan sus propios recursos y los funcionarios públicos se relacionan con ellos rindiéndoles cuenta de sus servicios (Durston, 2002).

Un interfaz ideal demandaría compromiso afectivo del agente externo con la comunidad (más que con personas específicas), una acción que favorezca el empoderamiento mediante un intercambio complementario (ambos actores tienen algo que aportar al otro) y alta confianza. Adicionalmente, debe existir una regulación de los conflictos.

Zambrano y Alfaro (2006) plantean que en Chile la emergencia de una nueva generación de políticas públicas constituye un escenario favorable a un enfoque que valora lo comunitario y el empoderamiento; sin embargo, la presencia de una institucionalidad caracterizada como tradicional, burocrática y centralizada obstaculiza en el plano operativo la lógica y espíritu de estrategias comunitarias.

En el análisis de la aplicación de la política social es central utilizar un enfoque relacional, toda vez que en el proceso de su implementación se juega la potencialidad de colaborar o no en los procesos de desarrollo (Roberts, 2001, Diciembre). En este sentido, la naturaleza de las relaciones establecidas entre los diversos actores involucrados en una comunidad podría potenciar los recur- sos de dicha comunidad, favoreciendo el ejercicio de una ciudadanía activa, o, por el contrario, fomentar relaciones que marginan o producen una ciudadanía desigual. Así, los agentes de un servicio público pueden favorecer procesos de empoderamiento o inhibirlos.

En esta perspectiva, en este estudio se analizan las relaciones entre las organizaciones comunitarias y los agentes públicos de dos municipios de la región de la Araucanía, evaluando en las trayectorias de estas organizaciones la capacidad de esas relaciones de aportar al empoderamiento organizacional y comunitario.

\section{Método}

Se empleó una metodología cualitativa de tipo descriptiva, en la que el foco de estudio fue el análisis de diferentes organizaciones comunitarias de dos localidades de la región de la Araucanía, las que se seleccionaron mediante un muestreo intencionado por criterios. Del mismo modo, se entrevistaron a funcionarios de ambos municipios.

\section{Participantes}

Primero se seleccionaron comunas de la región de la Araucanía con las que se tenía contacto previo con el municipio correspondiente, definiéndose como localidades a Quillem (comuna de Perquenco) y Puerto Saavedra urbano. Se seleccionaron las organizaciones de cada localidad de acuerdo a dos criterios: organizaciones que tuvieran una trayectoria de al menos un año y organizaciones de distinto tipo (e.g., deportivas, territoriales, juveniles). La cantidad de organizaciones a entrevistar en cada localidad se estableció una vez que se tuvo un diagnóstico actualizado de las organizaciones existentes y de su tipo de funcionamiento en cada una de las localidades. Las organizaciones cubiertas se describen en la Tabla 1.

En ambas comunas se entrevistaron además a funcionarios de los municipios. Las entrevistas fueron realizadas por estudiantes en práctica profesional y tesistas del área comunitaria, cubriendo en Quillem a casi el $80 \%$ de los funcionarios (quienes 
adicionalmente participaron en un taller de devolución y profundización de un diagnóstico organizacional) y en Puerto Saavedra, a cerca del $50 \%$ de los funcionarios.

\section{Instrumentos}

Las técnicas de recolección/producción de información empleadas fueron: entrevista semiestructurada, entrevista no estructurada, entrevista de grupo, observación participante y no participante y análisis de documentación (ver Tabla 2). Estas técnicas aportaron información respecto de las historias de las organizaciones, los tipos de vínculos internos y externos, sus lógicas de funcionamiento y los antecedentes de cada una de las localidades (historia local, ante- cedentes sociodemográficos, entre otros).

Las técnicas observación participante y no participante aportaron información respecto de los vínculos reales establecidos entre las organizaciones y sus miembros y agentes del municipio. Las técnicas de diagrama de Venn y el cuestionario generador de nombres aportaron información para el análisis estructural de redes. Las entrevistas a las organizaciones indagaron información respecto de la historia de la localidad y de la trayectoria de la organización, la relación entre dirigentes y municipio y la proyección del trabajo de la organización. Las entrevistas a los actores municipales indagaron sobre los tipos de relación establecidos con las organizaciones, la visión sobre las organizaciones locales y los enfoques

Tabla 1

Organizaciones Participantes del Estudio

\begin{tabular}{lcccc}
\hline Organización/Localidad & Quillem & Cantidad Integrantes & Puerto Saavedra & Cantidad Integrantes \\
\hline Juntas de Vecinos & 1 & 30 & 1 & 5 dirigentes \\
Comités de Vivienda & 1 & 46 & 1 & 60 familias \\
Club de Adulto Mayor & 1 & 15 & 1 & 30 \\
Organizaciones Juveniles & 1 & 16 & 1 & 5 directivas \\
Total & 4 & 107 & 4 & $\begin{array}{c}\text { estudiantes del Liceo } \\
\text { Reino de Suecia }\end{array}$ \\
\hline
\end{tabular}

Tabla 2

Técnicas por Localidad y Organización

\begin{tabular}{|c|c|c|c|c|c|c|c|c|}
\hline \multirow{3}{*}{ Técnicas } & \multicolumn{8}{|c|}{ Localidad } \\
\hline & \multicolumn{4}{|c|}{ Quillem } & \multicolumn{4}{|c|}{ Puerto Saavedra } \\
\hline & JJVV & $\begin{array}{l}\text { Adulto } \\
\text { Mayor }\end{array}$ & $\begin{array}{l}\text { Organización } \\
\text { Juvenil }\end{array}$ & $\begin{array}{l}\text { Comité de } \\
\text { Vivienda }\end{array}$ & JJVV & $\begin{array}{l}\text { Adulto } \\
\text { Mayor }\end{array}$ & $\begin{array}{l}\text { Organización } \\
\text { Juvenil }\end{array}$ & $\begin{array}{l}\text { Comité de } \\
\text { Vivienda }\end{array}$ \\
\hline Taller grupal & 3 & 3 & 3 & 2 & 3 & 3 & 3 & 3 \\
\hline Taller grupal & 1 & 1 & 1 & - & 1 & 1 & 1 & 1 \\
\hline $\begin{array}{l}\text { Observación } \\
\text { participante y } \\
\text { no participante }\end{array}$ & \multicolumn{8}{|c|}{ PERMANENTE A LO LARGO DEL PROCESO DE INVESTIGACIÓN } \\
\hline
\end{tabular}


de trabajo con las organizaciones. El equipo de investigación participó en actividades como: jornada de diagnóstico comunitario, festividades locales y reuniones de las organizaciones con el alcalde y el equipo municipal, en el caso de Quillem; y en la producción de un video con la reconstrucción histórica de la localidad, cuenta pública del alcalde y actividades recreativas, en el caso de Puerto Saavedra.

\section{Procedimiento}

La inserción en la comuna de Perquenco y la localidad de Quillem se realiza a partir del año 2004 y en Puerto Saavedra, desde el 2006. En ambos casos el contacto inicial se realiza con el Director de Desarrollo Comunitario. Posteriormente, se tomó contacto con los respectivos alcaldes, funcionarios municipales y organizaciones. En cada una de las comunas el equipo investigador participó en diversas actividades que permitieron tomar contacto con los actores locales. Luego se aplicaron las diversas técnicas de producción de datos y, posterior al análisis, se realizaron actividades de devolución-problematización de los resultados.

\section{Análisis de la Información}

La información susceptible de transcribirse y trabajarse en formato electrónico fue sometida a un análisis cualitativo con el software AtlasTi versión 4.04. El análisis se realizó siguiendo el enfoque de la teoría desde la base, utilizándose específicamente la codificación abierta y axial. Se integraron, además, otros datos como las observaciones de campo y diagramas de redes. El componente estructural de las redes se analizó mediante el software UCINET VI y desde Newtdraw se obtuvieron las representaciones gráficas de las redes estudiadas.

La credibilidad de los resultados se aseguró por la duración e intensidad de la observación-participación (entre los años 2005 y 2007) y por la triangulación de datos, de métodos y de investigadores. Se realizaron, también, chequeos y contra chequeos con las organizaciones participantes y funcionarios municipales.

\section{Resultados}

\section{Localidad de Quillem}

Características centrales de la dinámica comunitaria. Se observa una identidad local en la que predomina un fuerte sentimiento de exclusión en relación al resto de la comuna de Perquenco, con una conflictividad latente que se expresa con mayor intensidad en períodos eleccionarios de autoridades de la municipalidad, momento en que se hace evidente una fuerte división partidista centrada en las personas que postulan a ese cargo más que en la adscripción a partidos políticos. Es una comunidad que mantiene lazos por mucho tiempo, basados fuertemente en relaciones de parentesco. Los vecinos de la localidad deben enfrentar importantes dificultades económicas y de acceso a servicios y recursos. Con todo, se trata de una comunidad que tiende a solidarizar cuando eventos difíciles afectan a alguno de sus integrantes.

Estilo de funcionamiento en las organizaciones comunitarias. En sus trayectorias las organizaciones se caracterizan por un funcionamiento centrado en lograr objetivos de corto y mediano plazo, los que en gran parte se han remitido a conseguir recursos económicos. Las relaciones al interior de las organizaciones tienden a reproducir el esquema existente en otros espacios comunitarios, con alta concentración de responsabilidades y toma de decisiones en los dirigentes, quienes han logrado desarrollar cierto nivel de capital social que ponen al servicio de la organización. Asimismo, presentan un relativamente alto nivel de empoderamiento individual que, sin embargo, no logra traducirse en mayor empoderamiento organizacional.

La participación de los integrantes de las organizaciones se da principalmente en actividades que implican resultados en el corto o mediano plazo, que se asocian al acceso a recursos materiales o realización de actividades recreativas y culturales (e.g., fiestas patrias, fiesta de la primavera). Un actor facilitador de estas formas de participación es la escuela de la localidad, ya sea porque esta cede sus espacios físicos para 
la realización de actividades comunitarias o porque el equipo de profesores organiza algunas actividades abiertas a la comunidad.

En síntesis, los esquemas semiclientelares y paternalistas se reproducen al interior de las organizaciones, las que dependen en su trayectoria casi exclusivamente de las gestiones y logro de las directivas a nivel del municipio y otros programas.

En los vínculos de nivel vertical de las organizaciones predominan los nexos con el municipio, siendo la relación fundamental con el alcalde. El tipo de vínculo establecido es semiclientelar.

Relación municipio-comunidad. En una mirada histórica a las relaciones sostenidas por este municipio con la comunidad, se observa que cobra centralidad el estilo y tipo de rol del alcalde para definir la interfaz. Destaca como principal estilo de interfaz, desde la tipología de Durston (2002), el clientelismo paternalista pasivo:

Uno ve que el municipio es como el motor de la comuna y es el que tiene que solucionar todos los problemas (entrevista a funcionario municipal, párrafo 68 , código interfaz concepción "paternalista")

Esta situación comienza muy lentamente a modificarse en la medida que la gestión sostenida por el actual alcalde pone acento en la promoción y participación de las organizaciones, lo que ha exigido a los funcionarios ir modificando su estilo de interacción con la comunidad, así como a la comunidad, asumir un rol más activo.

El equipo municipal presenta un alto compromiso con la labor desarrollada por sobre el carácter político partidario, con un fuerte sentido de servicio público y, si bien tiene claro que debe avanzar en una relación menos asistencialista, ve en ello una difícil tarea porque percibe a la comunidad como muy necesitada de recursos y demandante de apoyo.

\section{Localidad de Puerto Saavedra}

Características centrales de la dinámica comunitaria. En el caso de Puerto Saavedra urbano, se aprecia una comunidad marcada por el terremoto vivido en la década de los sesenta, donde miran a un pasado de pros- peridad (antes del terremoto), en contraste con un presente de múltiples dificultades. Se advierte que, a partir de ese evento natural, la localidad ha sido objeto de una aguda y caótica secuencia de intervenciones desde distintos agentes y entidades movilizadas por diversos y disímiles intereses, que han tendido a reforzar una identidad local "sufriente". Además, en esta localidad cobra mayor relevancia el clientelismo político partidista:

\section{Pero el acostumbramiento y las prome- sas politicas de repente hacen que la gente no surja por si sola, es una cues- tión asi, porque los mal acostumbras... (entrevista a funcionario municipal, párrafo 27, código interfaz concepción "clientelar política")}

El énfasis de distintos organismos públicos y de ONGs en que la comunidad se organice para acceder a recursos ha incentivado la formalización de las organizaciones comunitarias. Sin embargo, esto ha generado quiebres en las formas de organización natural de las personas en la comunidad, asociacionismo en torno a la postulación de proyectos (uno de los principales focos de vinculación entre el municipio y la comunidad), restricción de la capacidad de autogestión y una comprensión limitada acerca de la participación.

Estilo de funcionamiento en las organizaciones comunitarias. Las organizaciones tienden a mostrar una visión utilitarista de la participación y organización comunitaria, la que está motivada por necesidades materiales concretas que pueden ser resueltas a través de las organizaciones.

Desde una perspectiva global, se aprecia que las organizaciones han vivido procesos de formalización, con el propósito central de obtener recursos de diversos programas estatales; de allí que se las perciba y valore esencialmente como un medio para acceder a recursos escasos más que como un espacio de desarrollo personal y comunitario.

En este contexto los líderes aparecen como mediadores entre el municipio y las bases, siendo por tanto relevante el capital social individual que estos hayan logrado desarrollar para alcanzar los propósitos de la organización. Así, el tipo de interacción 
prevaleciente es de distanciamiento de las bases y reproducción de un esquema asistencialista de relación.

Los dirigentes, particularmente de las organizaciones constituidas por adultos, presentan trayectorias prolongadas de participación (vinculadas a historias familiares de liderazgo), tendiendo a concentrar el poder social en la medida que asumen cargos en distintas organizaciones del mismo sector. Ellos son percibidos por los integrantes de las organizaciones como poco representativos, aunque se valora su experticia. Hay algunos dirigentes que se desempeñan además como funcionarios municipales, cuestión que agrava las desconfianzas por la superposición de intereses.

Los dirigentes valoran la posibilidad de servir, a través de su rol, a la comunidad, aunque viven sentimientos encontrados en la medida que, al mismo tiempo que responden a una vocación de servicio personal y familiar, experimentan una experiencia de sobrecarga.

Al realizar el análisis por tipo de organización, se observan similitudes entre ellas. En términos generales, las organizaciones se caracterizan por contar con redes predominantemente de baja densidad, cuyos vínculos tanto internos como externos están centralizados en una persona, la que generalmente es el dirigente y en ocasiones un agente municipal, con excepción de las organizaciones juveniles, las que parecen compartir mejor entre sus miembros las relaciones internas y externas.

Relación municipio-comunidad. En el período estudiado, la gestión municipal aparece altamente politizada, con una cultura organizacional marcada por la diferencia entre funcionarios de planta y funcionarios de confianza política del alcalde. En las relaciones que se sostienen con la comunidad se promueve el apoyo diferenciado a los dirigentes, mediado por favores personales y privilegios.

También se aprecia una gestión mediada fundamentalmente por la urgencia, siendo la comunidad definida desde la carencia, entendida no solo desde los recursos materiales sino, más bien, como en estado de necesidad permanente.
En este municipio se identifica la centralidad del rol del alcalde para definir el tipo de interfaz, predominando uno de tipo clientelar paternalista pasivo (Durston, 2002).

\section{Análisis Integrado de Resultados}

Aunque los diferentes actores locales valoran la organización y participación como elementos sustantivos para el desarrollo de la comunidad, las relaciones de interfaz sostenidas por ellos no favorecen procesos de empoderamiento organizacional ni comunitario. Se observa en ambas comunas que el capital social comunitario de tipo vertical y horizontal corresponde más bien al capital social individual de sus dirigentes más activos.

Una parte importante de las dificultades para que las organizaciones comunitarias obtengan logros de mayor impacto en sus objetivos está en su interior. La baja densidad de las relaciones, la centralización de los vínculos en la persona del o la presidenta de la organización, la baja renovación de liderazgos y el foco de la relación con el municipio y otros actores públicos puesto solo en la obtención de recursos materiales, constituyen obstáculos para que la comunidad acceda a recursos disponibles para mejorar sus condiciones de vida y aumentar su autonomía.

Desde ambos municipios predominan relaciones de interfaz del tipo clientelismo paternalista pasivo y de semiclientelismo. Estas relaciones de interfaz no facilitan el desarrollo de capacidades y de autogestión y la formación de capital social comunitario. Así, se retroalimenta un sistema local que mantiene relaciones de dependencia respecto del municipio, que dificulta el empoderamiento comunitario.

Es importante destacar, sin embargo, que en el municipio de la comuna de Perquenco, al momento del estudio, ocurrían cambios de relevancia en dirección a favorecer relaciones que fomentan la participación activa de la comunidad y un mayor protagonismo de las organizaciones, particularmente de la localidad de Quillem, cuestión mediada por un alcalde que valora e incentiva estos procesos. 
Discusión

Los resultados enfatizan la relevancia de las características del liderazgo ejercido por la autoridad del gobierno local como un factor clave en las trayectorias de las organizaciones, en sus relaciones con las organizaciones sociales y en el contexto en que los operadores públicos deben trabajar, particularmente en comunas con rasgos más bien tradicionales como en las que se insertan ambas localidades.

Asimismo, el enfoque predominante de vinculación con la comunidad encontrado en los agentes municipales aparece como un factor clave para incidir en la modificación de las relaciones clientelares, semiclientelares y asistencialistas que predominan en ambas localidades.

Lo anterior confirma los hallazgos de Durston et al. (2005) en relación al rol negativo que está teniendo el clientelismo partidario al interior de los municipios en su contribución al desarrollo de la comunidad, al no lograr avanzar hacia un estilo de relación "empoderador". Ello no quiere decir que falte voluntad en los agentes municipales para aportar al desarrollo de la comunidad, ya que los agentes estudiados están comprometidos con las organizaciones de la comunidad, aunque desde representaciones de tipo carencial. Es más bien el contexto clientelar más amplio en el que ellos trabajan el mayor obstáculo.

En relación a las barreras para favorecer el empoderamiento en el espacio comunitario (Maton, 2008), se puede destacar en el nivel de las organizaciones: debilidades en la estructura de las oportunidades de ejercer roles (los dirigentes centralizan las relaciones y decisiones), las características de los liderazgos (paternalistas o autocráticos) y las características de las actividades centrales desarrolladas por las organizaciones (orientadas a la recepción pasiva de recursos materiales).

Si bien el componente de acceso a recursos (Zimmerman, 2000) es prioritario en sectores con amplias dificultades sociales y económicas, los medios y las dinámicas que se configuran en torno a este proceso permiten o restringen el acceso a otros recursos también importantes. Para gran parte de las organizaciones en este estudio -en menor medida para las organizaciones de adultos mayores y de jóvenes- la organización y participación adquieren un valor estrictamente instrumental. Así, los componentes de participación crítica, dinámica democrática y vinculación con otras organizaciones -los otros componentes del empoderamiento organizacional y comunitario- están prácticamente ausentes en las organizaciones estudiadas.

Esta forma de funcionamiento, como ya ha sido descrita por Zambrano (2007), favorece que los programas sociales, particularmente los ejecutados en el espacio local, privilegien la relación/mediación con dirigentes por sobre la relación con la organización. Así, con frecuencia los líderes y dirigentes viven fuertes procesos de empoderamiento individual sin que ello redunde necesariamente en un empoderamiento organizacional y comunitario. El peligro de esta fórmula, así como la concentración del poder solo en la autoridad municipal y en los técnicos, es que se refuercen y perpetúen las relaciones clientelares. Este esquema relacional con frecuencia permite que las organizaciones cumplan el rol instrumental de acceder a recursos, pero no impacta la calidad de las relaciones, las capacidades ni la subjetividad de los involucrados.

A partir del análisis de los resultados, se puede plantear que en ambas localidades se requiere de procesos intencionados de fortalecimiento organizacional, así como de una modalidad de trabajo desde los agentes públicos que sea promotora del empoderamiento de actores locales. Sin lugar a dudas, parte importante de este escenario se vincula con el estilo de liderazgo que ejercen las autoridades municipales de turno, las que pueden favorecer las relaciones clientelares o, en contrapartida, favorecer en el municipio un rol de mayor promoción y participación de la comunidad. También se puede apreciar que un estilo más democrático por sí solo no garantiza condiciones de empoderamiento, puesto que la matriz cultural que atraviesan las relaciones favorece el poder dominación por sobre el poder potencia (Villasante, 2002). Procesos sistemáticos de evaluación crítica y participativa, así como la formación técnica especializada para el 
trabajo comunitario de los funcionarios municipales, aparecen como prioritarios.

En la experiencia de campo en la región de la Araucanía (Zambrano \& LeBlanc, 2008, Junio) se muestra que el estilo predominante en muchas organizaciones comunitarias es similar al descrito en este estudio, requiriéndose para favorecer el empoderamiento comunitario de un proceso de formación y acompañamiento de líderes y organizaciones para el ejercicio de un liderazgo democrático.

También es importante propiciar en el resto de la comunidad procesos participativos, siendo el desafío prioritario rescatar los intereses y valoraciones culturales de la comunidad para favorecer la puesta en relación de los actores locales en un contexto de convivencia. A partir de esto, se puede transitar lentamente a un tipo de participación más activa y reflexiva, mediante procesos educativos transversales (Zambrano, 2007).

Por otra parte, se puede concluir que los recursos endógenos centrales de las organizaciones son las características de los dirigentes, el grado de participación activa de los miembros cuando las iniciativas comprometen su interés, así como el capital social interno a la organización. Las organizaciones tienden, desde esta perspectiva, a conseguir al menos parcialmente sus objetivos, lo que refuerza la conveniencia de participar y actuar organizadamente, aunque ello se haga en forma más bien pasiva.

Finalmente, cabe referirse brevemente a las limitaciones como a las posibles proyecciones de este estudio. $\mathrm{Al}$ menos dos debilidades deben ser mencionadas: en primer lugar, el escaso énfasis en el componente histórico en el estudio de las organizaciones (para haber profundizado en la noción de trayectoria) y, en segundo lugar, no haber contemplado a otros informantes de la comunidad no integrados a organizaciones, con el propósito de haber tenido una visión más panorámica de las dinámicas comunitarias.

En el plano de las proyecciones sería conveniente estudiar trayectorias organizacionales positivas en alguna localidad que presente mejores niveles de empoderamiento comunitario, con el propósito de indagar en aquellos factores que se asocian a este proceso.

\section{Referencias}

Alfaro, J. (2000). Discusiones en psicología comunitaria. Santiago, Chile: Universidad Diego Portales.

Alfaro, J. \& Zambrano, A. (en prensa). Psicología comunitaria y políticas sociales en Chile. Psicología $\&$ Sociedad.

Barbero, J. \& Cortés, F. (2005). Trabajo comunitario, organización y desarrollo social. Madrid: Alianza.

Barreiro, F. (2002). Desarrollo desde el territorio: a propósito del desarrollo local. Cataluña: Instituto Internacional de Gobernabilidad de Cataluña. Extraído el 10 Octubre, 2003, de http://www. iigov.org/documentos/?p=3_0014

Blanco, I. \& Rebollo, O. (2002). El Plan Comunitario de la Trinitat Nova. Barcelona: Universitat Autónoma de Barcelona.

Boisier, S. (1999). ¿Desarrollo (local): ¿de qué estamos hablando? (Cuadernos Regionales $\mathrm{N}^{\mathrm{o}} 1$ ). Talca, Chile: Universidad de Talca.

Boisier, S. (2001, Noviembre). Crecimiento y desarrollo territorial endógeno: observaciones al caso chileno. Ponencia presentada en el Seminario Internacional Desarrollo Endógeno en Territorios Excluidos, Temuco, Chile.

Bustamante, G. (2003). Iniciativa de (etno) desarrollo endógeno-local: estudio de caso del capital social de la organización indígena mapuche Ayjarewe Xuf Xuf. Tesis no publicada para optar al grado de Magíster en Desarrollo Humano a Escala Local y Regional, Universidad de La Frontera, Temuco, Chile.

Carballeda, A. (2002). La intervención en lo social: exclusión e integración en los nuevos escenarios sociales. Santiago del Estero, Argentina: Paidós.

Craig, G. \& Mayo, M. (1994). Community empowerment. London: Zed Books.

De Paula, V. (2003). Estrategias de empowerment en trabajo social. Buenos Aires: Lumen Hvmanitas.

Dirven, M. (2001, Septiembre). Entre el ideario y la realidad: capital social y desarrollo agrícola, algunos apuntes para la reflexión. Ponencia presentada en la Conferencia En Busca de un Nuevo Paradigma: Capital Social y Reducción de la Pobreza en América Latina y el Caribe, Santiago, Chile.

Durston, J. (1999). Construyendo capital social comunitario: una experiencia de desarrollo comunitario en Guatemala [Versión electrónica] (Serie Políticas Sociales $\mathrm{N}^{\circ}$ 30). Santiago, Chile: Comisión Económica para América Latina y el Caribe.

Durston, J. (2002). El capital social campesino en la gestión del desarrollo rural: díadas, equipos, puentes y escaleras. Santiago, Chile: Comisión Económica para América Latina y el Caribe.

Durston, J., Duhart, D., Miranda, F. \& Monzó, E. (2005). Comunidades campesinas, agencias públicas $y$ clientelismos políticos en Chile. Santiago, Chile: LOM.

Flores, M. \& Rello, F. (2001, Septiembre). Capital social: virtudes y limitaciones. Ponencia presentada en 
la Conferencia En Busca de un Nuevo Paradigma: Capital Social y Reducción de la Pobreza en América Latina y el Caribe, Santiago, Chile.

Friedmann, J. (1992). Empowerment. The politics of alternative development. Oxford, Inglaterra: Blackwell.

García, M. (2005). El surgimiento de la cooperación entre actores que operan instrumentos de la política pública: un estudio de caso en el proceso de postulación del comité de vivienda Presidente Lagos de la comuna de Nueva Imperial al Programa de Fondos Solidarios Concursables del Ministerio de Vivienda y Urbanismo. Tesis no publicada para optar al grado de Magíster en Desarrollo Humano a Escala Regional y Local, Universidad de La Frontera, Temuco, Chile.

Gil, E. (2003). El poder gris: una nueva forma de entender la vejez. Barcelona: Grijaldo Mondadori.

Kliksberg, B. (1999). Capital social y cultura, claves esenciales del desarrollo. Revista de la CEPAL, 69, 85-102.

Krause, M. \& Jaramillo, A. (1998). Intervenciones psicológicas comunitarias en Santiago de Chile. Santiago, Chile: Pontificia Universidad Católica de Chile.

Lapalma, A. (2001). El escenario de la intervención comunitaria. Revista de Psicología Universidad de Chile, 10(2), 61-70.

Le Bossé, Y. \& Dufort, F. (2002). El empoderamiento de las personas y comunidades: otra forma de intervenir. En F. Dufort \& J. Guay (Eds.), Agir au coeur des communautés: La psychologie communitaire et le changement social (pp. 75-115). Laval, Canadá: Les Presses de l’Université Laval.

Lipszyc, C. (1993). Las mujeres y el poder ¿Podemos las mujeres transformar el sistema de poder? Feminaria, 11, 11-20.

Long, N. (1999). The multiple optic of interface analysis (working title). Austin, TX: University of Texas at Austin, Center for Latin American Social Policy. Extraído el 10 Octubre, 2003, de http://lanic.utexas.edu/project/etext/llilas/claspo/ workingpapers/multipleoptic.pdf

Llena, A. \& Ucar, X. (2006). Acción comunitaria: miradas y diálogos interdisciplinares e interprofesionales. En X. Úcar \& A. Llena (Coords.), Miradas y diálogos en torno a la acción comunitaria (pp. 1155). Barcelona: GRAÓ.

Marchioni, M. (2001). Comunidad y cambio social. Teoría y praxis de la acción comunitaria. Madrid: Popular.

Martínez, V. (2006). El enfoque comunitario. El desafío de incorporar a la comunidad en las intervenciones sociales. Tesis no publicada para optar al grado de Magíster en Psicología Comunitaria, Universidad de Chile, Santiago, Chile.

Maton, K. I. (2008). Empowering community settings: Agents of individual development, community betterment, and positive social change. American Journal of Community Psychology, 41, 4-21.

Moncayo, E. (2001). Evolución de los paradigmas y modelos interpretativos del desarrollo territorial [Versión electrónica]. Santiago, Chile: Instituto Latinoamericano y del Caribe de Planificación Económica y Social.

Montero, M. (1991). Acción y discurso: problemas de psicología política en América Latina. Caracas: Eduven.

Montero, M. (2003). Teoría y práctica de la psicología comunitaria: la tensión entre sociedad y comunidad. Buenos Aires: Paidós.

Montero, M. (2005). Introducción a la psicología comunitaria. Desarrollo, conceptos y procesos. Santiago del Estero, Argentina: Paidós.

Newbrough, J. R. (1992). Community psychology in the postmodern world. Journal of Community Psychology, 20, 10-25.

Pérez-Luco, R. (2003). Enfoque psicosocial ecológico: psicología comunitaria en La Frontera. En L. Rehbein (Ed.), Aportes y desafíos desde la práctica psicológica (pp. 371-400). Santiago, Chile: RIL.

Perkins, D. D. (1995). Speaking truth to power: Empowerment ideology as social intervention and policy. American Journal of Community Psychology, 23, 765-794.

Plough, A. \& Olafson, F. (1994). Implementing the Boston Healthy Start Initiative: A case study of community empowerment and public health. Health Education Quarterly, 21, 221-234.

Rappaport, J. (1981). In praise of paradox: A social policy of empowerment over prevention. American Journal of Community Psychology, 9, 1-25.

Rappaport, J. (1987). Terms of empowerment/ exemplars of prevention: Toward a theory for community psychology. American Journal of Community Psychology, 15,121-148.

Roberts, B. R. (2001, Diciembre). Las nuevas políticas sociales en América Latina y el desarrollo de ciudadania: una perspectiva de interfaz. Ponencia presentada en el Taller Agencia, Conocimiento y Poder: Nuevas Direcciones, Wageningen, Holanda. Extraído el 10 Octubre, 2003, de http://lanic.utexas.edu/project/etext/llilas/claspo/ workingpapers/unaperspective.pdf

Rowlands, J. (1997). Questioning empowerment: Working with women in Honduras. Oxford, Inglaterra: Oxfam.

Rozas, G. (1995). Psicología comunitaria en el desarrollo local y regional. Revista de Psicología Universidad de Chile, 5, 46-64.

Saavedra, C. (2005). Aproximación al desarrollo actual de la psicología comunitaria desde el análisis de las prácticas que ésta construye en el campo de la intervención social. Tesis no publicada para optar al grado de Magíster en Psicología Comunitaria, Universidad de Chile, Santiago, Chile.

Serrano, C. (2005). La política social en la globalización. Programas de protección en América Latina (Serie Mujer y Desarrollo No 70). Santiago, Chile: Comisión Económica para América Latina y el Caribe.

Serrano-García, I. \& López, G. (1994). Una perspectiva diferente del poder y el cambio social para la comunidad. En M. Montero (Coord.), Psicología social comunitaria. Teoría, método y experiencia (pp. 167-209). Guadalajara: Editorial Universidad de Guadalajara.

Shein, E. (1998). Consultoría de procesos. Volumen 2. Ciudad de México: Editorial Addison-Wesley Iberoamericana.

Silva, C. \& Martínez, M. L. (2004). Empoderamiento: proceso, nivel y contexto. Psykhe, 13(2), 29-39.

Taliferro, M. B. (1991). The myth of empowerment. 
The Journal of Negro Education, 60, 1-2.

Vásquez, C. (2004). Refortalecimiento: un debate con el empowerment. Revista Interamericana de Psicología, 38(1), 39-49.

Vásquez-Barquero, A. (1999). Desarrollo, redes e innovación: lecciones sobre desarrollo endógeno. Madrid: Ediciones Pirámide.

Villasante, T. (2002). Proceso para la creatividad social. Madrid: El Viejo Topo.

Zambrano, A. (2007). Criterios de intervención en estrategias de empoderamiento comunitario: la perspectiva de profesionales y expertos de la intervención comunitaria en Chile y España. Tesis no publicada para optar al grado de Doctor en Psicología Social, Universidad de Barcelona, España.

Zambrano, A. \& Alfaro, J. (2006). Desarrollo y estado actual de la psicología comunitaria en Chile: trayectorias, tensiones y perspectivas. Revista de Psicología Universidad de Valparaíso, 3, 11-22.

Zambrano, A. \& LeBlanc, L. (2008, Junio). Evaluación de necesidades y recursos para la implementación de una estrategia de formación y acompañamiento de líderes interesados en la prevención comunitaria de la drogodependencia. Ponencia presentada en la $2^{\text {nd }}$ International Conference on Community Psychology, Lisboa, Portugal.

Zimmerman, M.A. (1995). Psychological empowerment: Issues and illustrations. American Journal of Community Psychology, 23, 581-599.

Zimmerman, M. A. (2000). Empowerment theory: Psychological, organizational and community levels of analysis. En J. Rappaport \& E. Seidman (Eds.), Handbook of community psychology (pp. 43-63). New York: Kluwer Academic/Plenum Publishers.

Fecha de recepción: Febrero de 2009.

Fecha de aceptación: Septiembre de 2009. 